\title{
Educación y formación para la ciudadanía en y desde la universidad: aportes para el debate*
}

\author{
Margarita Benjumea-Pérez*** \\ Alejandro Mesa-Arango ${ }^{* * * *}$ \\ Universidad de Antioquia, Colombia \\ https://doi.org/10.15446/frdcp.n20.91013
}

\section{Resumen}

Este artículo da cuenta de algunas elaboraciones conceptuales y reflexiones alcanzadas en la investigación "La formación ciudadana en el contexto universitario de la UdeA -análisis de estrategias para su resignificación y potencialización desde la cotidianidad en el campus-", cuyo propósito fue identificar, visualizar y comprender realidades que acontecen en este escenario universitario frente al fenómeno de la formación ciudadana $\mathrm{y}$, los dispositivos formativos que se despliegan en pro de este propósito. Este ejercicio investigativo se realizó desde una perspectiva cualitativa, un enfoque hermenéutico

\footnotetext{
* Artículo recibido: 16 de octubre de 2020 / Aceptado: 8 de febrero de 2021 / Modificado: 22 de febrero de 2021. Este artículo es producto de una investigación financiada por el Fondo del primer proyecto de la Universidad de Antioquia, 2013-2015.

** Doctora en Educación, línea Formación Ciudadana por la Universidad de Antioquia, Colombia. Profesora titular de la Universidad de Antioquia, Colombia. Miembro del grupo de investigación "Cultura somática". Correo electrónico: margarita.benjumea@udea.edu.co (iD) https://orcid.org/0000-0002-6835-4273

**** Doctor en Educación, línea Formación Ciudadana por la Universidad de Antioquia, Colombia. Profesor titular de la Universidad de Antioquia, Colombia. Miembro del grupo de investigación "Comprender". Correo electrónico: alejandro.mesa@udea.edu.co (iD) https://orcid.org/0000-0001-5637-2645
}

Cómo citar

Benjumea-Pérez, M. y Mesa-Arango, A. (2021). Educación y formación para la ciudadanía en y desde la universidad: aportes para el debate. FORUM. Revista Departamento Ciencia Política, 20, 86-109. https://doi.org/10.15446/frdcp.n20.91013 
e investigación-acción como estrategia metodológica. Que busca además, motivar a la reflexión, acción y compromiso con esta tarea; y, consecuente con ello, diseñar e implementar una estrategia de acción complementaria al desenvolvimiento de vida académica en, con y desde miembros de esta comunidad educativa - propósito alcanzado-.

Palabras clave: educación superior; formación ciudadana; educación para la ciudadanía; estrategias formativas; formación política.

\title{
Education and Training for Citizenship in and from the University: Contributions to the Debate
}

\begin{abstract}
This article gives an account of some conceptual elaborations and reflections reached in the study "Citizen Training in the University Context of the UdeA - Analysis of Strategies for its Resignification and Potentialization from Daily Life on Campus", whose purpose was to identify, visualize and understand realities that occur in this university setting in the face of the phenomenon of civic education and the training devices that are deployed for this purpose; also seeking to motivate reflection, action and commitment to this task; and, consequently, to design and implement a complementary action strategy to the development of academic life in, with and from members of this educational community - purpose achieved-. Investigative exercise of qualitative perspective, hermeneutical approach and action research as a methodological strategy.
\end{abstract}

Keywords: higher education; citizen training; education for citizenship; training strategies; political formation.

\section{Introducción}

La relación educación, formación y ciudadanía ha contado con un renovado interés en las últimas dos décadas a juzgar por los textos que con respecto al tema se han generado desde instancias como Naciones Unidas, Cumbres de jefes de Estado, reuniones de sectores productivos y educativos y asociaciones de intelectuales, entre otras, a los que se suman investigaciones y reflexiones desde diversos campos. Y no es para menos, dada la emergencia de la globalización, la disolución de las naciones-Estado y las preocupaciones comunes que se comparten en ámbitos que van desde lo local hasta lo universal, como sucede, por 
ejemplo, con los derechos humanos, el derecho internacional humanitario y el cuidado del medio ambiente. De hecho, los grandes proyectos sociales que implican cambios de diversa índole ameritan que se tengan en mente algunos valores ciudadanos que trascienden las fronteras de los países, los cuales es necesario promover y desarrollar de manera intencionada, al menos desde las instituciones educativas en los Estados democráticos.

En este artículo se expondrán algunos de los lineamientos conceptuales y teóricos que animaron y sustentaron la discusión emergente en el desarrollo de la investigación "La formación ciudadana en el contexto universitario de la UdeA -análisis de estrategias para su resignificación y potencialización desde la cotidianidad en el campus-", llevada a cabo en la Universidad de Antioquia (2013-2015); la cual partió, justamente, de preocupaciones por la relación educación, formación y ciudadanía, en un ámbito que, como el universitario, ha contado con un desarrollo menor si se lo compara con lo que ha venido sucediendo en la educación básica y media. Debates que siguen siendo actuales y asisten hoy a las reflexiones del quehacer universitario.

\section{Puntos de partida}

La ciudadanía, más allá de toda tipología, es un artificio, una construcción humana determinada por la visibilización de sujetos, otrora caracterizados por ser "menores de edad", en sentido kantiano; es decir, por la falta de reconocimiento de sus facultades de pensamiento autónomo - pensar por sí mismo-, pensamiento coherente - pensar de acuerdo consigo mismo- y pensamiento ampliado - capacidad de ponerse en el lugar del otro-. Este último, justamente, tuvo su antecedente más importante en la antigua Grecia en lo que hoy se reconoce como el germen de la democracia moderna, expresada en el interés por los asuntos de la polis, mediante la libertad de actuar y la de decir, que, histórica y lentamente se ha ido ampliando con aquellos grupos y comunidades a quienes se les ha concedido o que han conquistado el derecho a aparecer en el espacio público.

Hablar de ciudadanía refiere a una construcción colectiva cuyo desarrollo histórico, parafraseando a Hannah Arendt, es la historia de la libertad en el espacio público, en medio de la aparente paradoja de la igualdad y la diferencia que caracteriza a los seres humanos. Igualdad porque aparecemos en un mismo espacio y diferencia porque dicho espacio se nos aparece de formas diversas que no resisten la clasificación de mejores o peores, sino que son simple y llanamente humanas (Arendt, 2008). En efecto, la historia de la ciudadanía es una historia tejida por agentes que, como se ha dicho, hacen su aparición en el mundo humano, mediante dos características fundamentales: 1) la acción o principio de natalidad en el que los seres humanos despliegan su capacidad creativa 
para resolver los asuntos de la comunidad; y 2) el discurso o principio de pluralidad, mediante el cual se ponen en diálogo todos los puntos de vista acerca de los hechos o los acontecimientos de dicha comunidad, para generar sentido común.

Conforme se ha ampliado históricamente la condición de ciudadanos, los Estados han desarrollado diversas maneras de integrar a los nuevos ciudadanos a la comunidad, de tal forma que la historia y el territorio compartido, así como los acuerdos a los que ha llegado la sociedad les sean dados a conocer, en procura de que dicho conocimiento los prepare para el ejercicio que de ellos se espera. En consecuencia, la relación educación y ciudadanía se ha establecido como un mecanismo de integración de los "recién llegados"1 a la comunidad.

Con todo, el clásico trabajo de Marshall y Bottomore (2005), publicado por primera vez en 1950, Ciudadanía y clase social, en el que el ciudadano deviene en sujeto jurídico de los derechos civiles, políticos y económicos, cuyos respectivos desarrollos se han logrado en los siglos XVIII, XIX y XX, continúa alimentando las discusiones tanto acerca de la ciudadanía en sí misma como de su relación con la educación y la formación. Es un hecho que la educación para la ciudadanía se reconoce como un asunto de vital importancia en los países en general, tal y como lo evidencia la gran cantidad de literatura especializada en la que se da cuenta de los avances, los esfuerzos, las inquietudes y los retos que ella comprende.

En efecto, solo por citar algunos ejemplos de publicaciones especializadas, se tiene el Journal of Moral Education, que se ha ocupado de la relación entre educación para la ciudadanía y desarrollo moral; el Journal of Social Education, que ha puesto su énfasis en la relación entre ciudadanía y cultura; el Journal of Beliefs \& Values, en los valores y la religión; y el Comparative Education Review, que ha enfatizado en los estudios comparados acerca de la ciudadanía y la educación para la ciudadanía entre diferentes países. Muchas publicaciones como estas, así como trabajos de investigación e informes de trabajos de maestrías y doctorados, han profundizado en la necesidad imperiosa de que los Estados y sus sistemas educativos, dediquen recursos y esfuerzos a la preparación para el ejercicio ciudadano de cada uno de sus miembros.

\section{Educación y formación}

Al tema de la formación de ciudadanos se hace referencia casi indistintamente como formación ciudadana, educación ciudadana, educación para la ciudadanía, educación

1. Recién llegado, es el modo en que se refiere Hannah Arendt a los niños y jóvenes a quienes la generación anterior tiene la obligación de formar (Arendt, 2007, p. 57). 
cívica y aun se le puede encontrar como cultura ciudadana ${ }^{2}$. La gama y las orientaciones son mucho más amplias si se atiende a los nombres que reciben las cátedras institucionales relacionadas en la educación superior. Algunos de estos nombres son: convivencia ciudadana, ética y valores, historia socioeconómica de Colombia, socioantropología, formación ciudadana y constitucional, trabajo comunitario, constitución política, competencias sociales, problemas de contexto, etcétera. A las que habría que agregar los espacios de formación social y humana que tienen un lugar en el plan de estudios y que se relacionan con el ejercicio ciudadano desde la profesión respectiva.

La referencia que hicieron ya hace algunos años Santana, Bogoya y Rojas (2005) sigue siendo de utilidad no solo para comprender las modalidades mediante las cuales se vinculan educación y ciudadanía, referidas de modo general a procesos intencionados institucionalmente, sino también para establecer una diferencia de la educación con la formación que ha venido promoviendo la línea de formación del doctorado en educación en ciencias sociales y humanas de la Universidad de Antioquia (Colombia). De acuerdo con los autores mencionados la relación de educación y ciudadanía en la educación formal se realiza con fundamento en tres mediaciones. La primera, educación sobre la ciudadanía: basada en los contenidos que deben aprender los estudiantes en las cátedras relacionadas con el tema; su orientación está más ligada a la denominada instrucción cívica en el marco de un proceso en el que el conocimiento acerca de los derechos y los deberes, así como de la organización del Estado son considerados una condición previa para el futuro ejercicio de la ciudadanía. La segunda, educación a través de la ciudadanía: basada en el aprender haciendo; en esta modalidad se incluyen ejercicios como el del Gobierno escolar, por medio del cual se espera que los estudiantes aprendan acerca de la organización del Estado y de los derechos y los deberes de los ciudadanos, mediante la representación del funcionamiento de algunos entes del Estado en las escuelas y los colegios o el ejercicio efectivo de representación en consejos, comités o grupos que tienen alguna injerencia en la toma de decisiones. La tercera, educación para la ciudadanía: que alude al aprendizaje de contenidos, el desarrollo de procesos cognitivos, la definición y la asimilación de valores, la génesis de actitudes para el desempeño de los roles que les demande la sociedad y la participación responsable y solidaria en los asuntos públicos. En esta modalidad se incluyen los servicios de voluntariado en la educación básica y los denominados de docencia-servicio, de amplia difusión en la educación para la ciudadanía en la educación superior.

2. Cultura ciudadana fue como se denominó al conjunto de actividades educativas encaminadas a promover el cambio de actitudes, prácticas y costumbres de los ciudadanos de la capital de la República de Colombia, durante los dos periodos de administración del alcalde Antanas Mockus (1995-1997, 2001-2003). 
Como se ve, la primera de las modalidades (sobre) se basa en la información que recibe el estudiante en el aula; la segunda (a través de), en la representación de la organización del Estado en la institución escolar; y la tercera (para), en la realización de actividades generalmente extramurales mediante las cuales se generan y promocionan valores ciudadanos. No obstante, la distinción que hacen Santana, Bogoya y Rojas (2005), la educación para la ciudadanía es el vocablo más ampliamente difundido y se usa indistintamente para hacer referencia a las tres modalidades. Una tipología que realiza Bolívar (2007), dos años más tarde, guarda similitud con la de esos autores, la cual es menester también tener en cuenta.

La educación sobre la ciudadanía corresponde en la tipología de Bolívar (2007) a la educación para la ciudadanía en el currículo formal con énfasis en los conocimientos adquiridos y actividades que se llevan a cabo en el aula; la educación en ciudadanía corresponde a la educación para el currículo informal por medio de las actividades y estrategias dentro de la institución, tales como la del Gobierno escolar y la participación en la toma de decisiones; y la educación para la ciudadanía, corresponde a la educación intencionada para este propósito en el currículo no formal, mediante el desarrollo de actividades conjuntas entre la escuela y la comunidad.

En la postura de Santa Cruz (2004), la educación para la ciudadanía busca que, dotando a los estudiantes con los conocimientos, así como con los principios, las capacidades, las normas, las herramientas y los valores que orientan el ejercicio de la ciudadanía, en el futuro, ellos ejerzan como ciudadanos actores, capaces de adaptarse a un entorno global en constante cambio. Para el caso de la educación superior se pretende que el ejercicio ciudadano en los estudiantes se dé al unísono con su formación en la universidad. En contraste con la perspectiva de "resultado", orientado en general por el estatus jurídico de ciudadanía, Bolívar (2007) plantea una mirada de corte mucho más amplia en la que más que un estatus, la ciudadanía constituye un proceso cuyo aprendizaje debe darse de modo permanente en el espacio público.

\section{Antecedentes de investigación sobre la relación educación, formación y ciudadanía en la universidad}

Si bien la normatividad colombiana deja claras las líneas de obligatoriedad para implementar procesos de educación para la ciudadanía en los niveles de educación preescolar, básica y media, no sucede así para la etapa de la educación superior. Pareciera que se da por entendido que los procesos desarrollados frente a esta temática en los niveles inferiores, fuesen suficientes, y que allí el ciudadano adquiriría la solvencia necesaria para su interacción y responsabilidad frente al proyecto de sociedad. Nada más lejano a la realidad porque la profunda brecha entre el ideal trazado de forma magistral en la Carta Constitucional y la realidad, se vive en muchos espacios de la cotidianidad - que no escapan a las dinámicas 
universitarias-; realidad que muestra claramente que se han descuidado las acciones en esta dirección. Es a esto a lo que se refiere Martínez (2006, p. 92) cuando señala que "al margen de declaraciones y documentos, el mundo universitario está apartado de estas preocupaciones y tiene cierto recelo para realizar planteos acerca de la formación ciudadana y en valores democráticos como algo inherente a la formación universitaria”.

No se encuentra tal despliegue de reflexiones e investigaciones acerca de la educación para la ciudadanía en el ámbito universitario, dado que esa suerte de sinonimia entre el menor y el escolar difícilmente podría aplicarse al joven y al universitario. No obstante, el tema ha ido tomando el carácter de emergente en los últimos años, debido a que las reflexiones exploradas identifican las principales inquietudes que el tema despierta. De manera sintética, esas inquietudes se han expresado en tres sentidos: en reflexiones acerca del papel de la universidad como escenario para la formación ciudadana; en informes de investigación en que los estudiantes universitarios forman parte de la categoría general de "jóvenes"; en informes de investigación con jóvenes universitarios propiamente.

La primera de esas tendencias, como es lógico, reclama la necesidad de recuperar el compromiso cívico de la universidad. En ella se pueden encontrar trabajos como los de Del Basto (2006), quien señala que la educación superior debe, justamente, recobrar su telos de espacio público en el que tienen ocasión la formación moral y la formación ciudadana, trascendiendo así su papel técnico-científico y, en coincidencia con Wilhite y Silver (2005), quienes afirmaron que la pérdida del rumbo en la misión cívica de la universidad se evidencia en su falta de compromiso para avanzar en torno a la construcción de lo público como bien común, mediante el desarrollo de las habilidades cívicas de los estudiantes sin que ello riña, como se piensa comúnmente, con la calidad técnica y científica. Por su parte, Englund (2002) afirma que el espacio universitario es privilegiado para el debate democrático teniendo en el pluralismo su condición primordial.

En sintonía con los anteriores postulados se encuentra el estudio de Benjumea et al. (2015) -fundamentos que alimentan este escrito-, develando una serie de dispositivos formativos que se despliegan en distintos espacios de la vida universitaria con intencionalidades de formación ciudadana; proponiendo, a su vez, una estrategia de acción creativa: "Re-Creándonos: una apuesta a la construcción de ciudadanías en y desde la $\mathrm{U}^{\prime \prime}$, surgida de las necesidades, los intereses, los gustos y las posibilidades

\footnotetext{
3. Re-creándonos es una iniciativa surgida a finales de 2012, propuesta por un pequeño grupo de actores del Instituto Universitario de Educación Física y Deportes de la Universidad de Antioquia, Colombia. Desde esa fecha hasta hoy, desarrolla y despliega todas sus acciones desde la Ciudadela Robledo de la Universidad. Contacto: re.creandonos@udea.edu.co
} 
de la comunidad educativa que en ella intervienen, como propósito de resignificación y potenciación de la formación ciudadana en la cotidianidad del campus universitario.

Y es que la universidad, y en particular la universidad pública, se configura como un espacio para la multiculturalidad, la diferencia; escenario de una amplia gama de pensamientos y acciones no todos convergentes en cuanto intereses, posibilidades y modos de actuación e interacción; hechos que aunque invaluables en su riqueza y posibilidades, la hacen igualmente vulnerable a permanentes tensiones de diferente índole y matices, permeable a todo tipo de problemáticas sociales que reflejan la realidades de la sociedad en que se inscribe; teniendo -infortunadamente- mayor eco ante la colectividad, aquellos eventos que se constituyen en puntos desfavorables por la fuerza negativa que alcanzan en los imaginarios y las representaciones sociales que frente a la universidad tiene una buena parte de la sociedad.

En la segunda tendencia, aquella que subsume a los universitarios en la categoría general de "jóvenes", pueden incluirse investigaciones como la de Del Río y Rizzini (2010) sobre factores y agentes sociales que inciden en el compromiso cívico de jóvenes comprometidos en Río de Janeiro y Ciudad de México; la de Warwick (2008), que relaciona la participación en la educación para la ciudadanía con la "agenda de la voz de los jóvenes" en Inglaterra; la de Haste y Hogan (2006), en el Reino Unido, que indaga por la relación entre la moral y la política; la de Eyler, Giles y Braxton (1997), en la que se explora la aplicación de las "etapas" de juicio reflexivo a la solución de problemas en los campos de práctica que enfrentaron estudiantes de colegios y universidades inscritos en "programas de docencia-servicio" y, finalmente, la de Alvarado et al. (2008), en que examinan el desarrollo de la subjetividad política en jóvenes participantes del proyecto "Jóvenes constructores de paz" en cuatro regiones de Colombia.

En esta tendencia, igualmente, puede visualizarse el proyecto "Re-creándonos", estrategia que desde 2013 hasta hoy, 2020, se configura en un espacio vivo transversalizado por un permanente ejercicio investigativo de reflexión-acción, en torno a los acontecimientos que les suceden a los jóvenes en su paso por la vida universitaria. Desde sus inicios, se proyecta activamente como un espacio de construcción social, política y profesional de todos, para todos y con todos los miembros de la comunidad universitaria de la UdeA, aportando con estas acciones a un mejor estar en los espacios que cohabitan, bajo una perspectiva de liderazgo y corresponsabilidad social universitaria, que abogue por su formación como sujetos políticos; aportando, de este modo, a la formación para su actuación, participación y corresponsabilidad en el escenario de lo social, de lo común y de lo público.

En la tercera tendencia, aquella en la que se agrupan las investigaciones realizadas con grupos de estudiantes universitarios propiamente, pueden incluirse los trabajos de Lozano y Alvarado (2011) con un grupo de estudiantes en quienes se exploraron conceptos, 
como política, ciudadanía y democracia; la de Quintero (2006), en que se exploró el concepto de justicia que subyace a las justificaciones de un grupo de jóvenes universitarios en comparación con un grupo de jóvenes desplazados; la de Tonon (2009) sobre la percepción de jóvenes universitarios del pregrado en Ciencia Política de la Universidad de Matanza en Buenos Aires; y la de Cuello (2010) sobre la relación existente entre la formación profesional y la pertenencia a la universidad con la comprensión de temas políticos.

En la misma perspectiva, se han desarrollado investigaciones en la Universidad de Antioquia, entre las que se cuentan la de Sandoval et al. (2012), en que se exploran las representaciones, los conocimientos y los juicios acerca de lo político en estudiantes de la Universidad de Antioquia; la tesis doctoral de Mesa (2011), en la que se indagó por el juicio reflexivo de un grupo de estudiantes de la Escuela de Microbiología de la Universidad de Antioquia, y la de Pimienta et al. (2015), quienes identificaron la relación y las interacciones del discurso y del proyecto político de universidad con la constitución de subjetividades políticas en estudiantes de cuatro programas de la Universidad de Antioquia. Esta última investigación es particularmente importante si se tiene en cuenta lo que podríamos llamar "el ascenso" de la subjetividad política como categoría de análisis que viene siendo desarrollada, además, por la Universidad de Manizales en las líneas de maestría y de doctorado relacionadas con la línea de estudio de niñez y juventud.

Entre los temas afines a la formación ciudadana que se contemplan con respecto a la investigación en estudiantes universitarios, se encuentran también, el de los movimientos estudiantiles y el de las percepciones sobre asuntos políticos. Como una respuesta al prejuicio de que los estudiantes universitarios se mostraban apáticos con respecto a la participación, se presentaron en las I Jornadas Internacionales sobre Educaciones y Política, llevada a cabo en Medellín, el $1^{\circ}$ y el 2 de noviembre de 2013, temas relacionados con la participación y el compromiso de los estudiantes universitarios en la coyuntura de la Reforma a la Ley 30 de 1992, que regula el sistema de educación superior.

Este último aspecto, aunque ligado a los movimientos estudiantiles de varias universidades en el país, ha marcado, sin duda, un punto de inflexión con respecto al prejuicio mencionado, toda vez que se constituyó en una fuente inagotable de actividades de información y de movilización, las cuales fueron consideradas por algunos estudiantes entrevistados en la investigación de Pimienta et al. (2015) como actividades de aprendizaje ciudadano no institucionalizadas, es decir, no pertenecientes al currículo formal.

Del mismo modo, otros espacios de disertación e interacción en torno a estas temáticas que la investigación de Benjumea et al. (2015) propició con comunidades estudiantiles y de otros estamentos de la Universidad de Antioquia, generaron nichos para ahondar en las percepciones que estos universitarios tienen con respecto a problemáticas que alteran su 
relación, compromiso, nivel de pertenencia con la universidad y el interés de participar activamente en diferentes escenas y escenarios de la vida universitaria. Desde allí pudo develarse que muchos de ellos consideran que estas "sensaciones y decisiones" de aislamiento en la participación de muchas de las dinámicas de la universidad; parten de los sentimientos de miedo, molestia, incapacidad, desconocimiento, impotencia, inseguridad e indiferencia que han tenido con respecto a situaciones que alteran la "normalidad" en la universidad desde que llegaron a ella; sensaciones que según ellos, son producto del "desconocimiento", "de no saber cómo participar", por ello, prefieren alejarse "porque tienen miedo" o "no les gusta la violencia", algunos creen que "hacen más por la universidad quedándose al margen".

Otra perspectiva que mostró este estudio, y que sigue siendo manifiesta en otros espacios investigativos actuales, revela que -a razón de este tipo de situaciones-y sumado a ello su condición económica, "la gran mayoría quieren acabar pronto su carrera"; pareciera no quedar más opción que centrar el paso por la universidad en lo académico, en el desarrollo de los contenidos de las áreas disciplinarias para obtener su título profesional pronto para salir a laborar. Asimismo, emergió de manera palpable "la vulnerabilidad de los recién llegados" como otra arista problemática de la cotidianidad universitaria, pues según los mismos jóvenes, "son vulnerables y manipulables" a pregones ideológicos que seducen y "convencen" de manera más próspera a estos jóvenes, "adoctrinándolos" en unos modos de pensamiento y acción que los persuade de "que el Estado y la universidad como representación estatal, son responsable de todos sus problemas, y tienen la posibilidad y obligatoriedad de resolvérselos. ¡solo hay que saber reclamarlo!”. Y desde allí, emerge otro sentido de corresponsabilidad, responsabilidad social y actitudes con, para, desde y hasta, en contra de la universidad.

\section{Educación, formación, ciudadanía y universidad}

La concienciación de la pertenencia del ciudadano a una determinada comunidad, pasa por la necesidad de implementar procesos mediante los cuales sus miembros acogen a los "recién llegados" con el compromiso de formarlos alrededor de los valores que comparten, de los derechos que les asisten y de los deberes que se espera que cada miembro cumpla y que vienen a constituirse en los derechos de los demás. Estos procesos están agenciados por la familia, el vecindario, la Iglesia y los medios de comunicación, entre otros; pero es claro que las expectativas sobre los procesos de inclusión de la sociedad moderna residen en la escuela, que funge como un primer espacio de aparición ${ }^{4}$, en el marco de la promoción de la homonimia.

4. El espacio de aparición de los ciudadanos, de acuerdo con Arendt (2008), es el lugar en que discuten, debaten y deliberan sobre los asuntos de la comunidad. 
La escuela es el primer espacio público para la socialización de los derechos humanos y para evitar que las diferencias se tornen en impedimentos para el ejercicio de la libertad (Alfaro, citado en Magendzo, 2004) y, en este sentido, sus esfuerzos se encaminan a tratar de garantizar a todos los niños y los jóvenes la formación en los conocimientos, las actitudes, los valores, los principios y los comportamientos necesarios para su inclusión en la sociedad.

La formación ciudadana en la educación superior, específicamente, requiere un conjunto de saberes, actuaciones y formas de pensar que contribuyan a la visibilización de los estudiantes en el espacio público; es decir, que la formación ciudadana de estudiantes universitarios debe hacer posibles los diferentes espacios de encuentro mediante la acción y el discurso entre pares. De este modo, el estudiante, además de ser formado como ciudadano, participa en la formación de otros, mediante su renuncia a la coacción, a fin de que cada uno pueda mostrarse tal cual es. El énfasis en la ciudadanía en el proyecto de investigación en el que se enmarca esta reflexión, tiene que ver justamente con la complementariedad entre lo político y lo jurídico en la formación ciudadana de los estudiantes universitarios.

Tal y como lo señalan Mesa y Benjumea (2011), las formas en que se ha abordado la educación para la ciudadanía en la educación superior están relacionadas estrechamente con las utilizadas en la educación básica y media, sin que resulte tan claro el papel que deben desempeñar las universidades en la formación ciudadana de sus estudiantes. De hecho, la instrucción cívica - referida a ese proceso de conocimiento de contenidos como los derechos y los deberes, condición previa para un adecuado ejercicio de la ciudadanía-, los mecanismos de participación y la forma de organización del Estado habitan lugares comunes.

Los programas de voluntariado, los servicios de asistencia y los servicios sociales, bajo la forma de alfabetización, de algún modo intentan reproducirse en la universidad por medio de los mismos programas de voluntariado y de prácticas profesionales mediante las cuales se espera que los estudiantes pongan su conocimiento al servicio de comunidades vulnerables. Sin embargo, tratándose de estudiantes universitarios es preciso asumirlos como mayores de edad, en el sentido en que Kant utiliza este término, teniendo como espacio de encuentro a la universidad, lo cual cobra aún mucho más sentido en la universidad pública-estatal en que una de las "competencias ciudadanas" debería ser el juicio crítico y su libre expresión; es decir, que la universidad en lugar de acciones y estrategias se configura en sí misma como un "espacio de aparición”. 


\section{Las competencias ciudadanas en contextos educativos}

El artículo 41 de la Constitución Política de Colombia de 1991 determinó que:

... en todas las instituciones de educación, oficiales o privadas, serán obligatorios el estudio de la Constitución y la instrucción cívica. Asimismo, se fomentarán prácticas democráticas para el aprendizaje de los principios y valores de la participación ciudadana. El Estado divulgará la Constitución.

Ley 1013 de 2006, artículo $1^{\circ}$ modificó el literal a) del artículo 14 de la Ley 115 de 1994, así:

a) El estudio, la comprensión y la práctica de la Constitución y la instrucción cívica, será materializada en la creación de una asignatura de Urbanidad y Cívica, la cual deberá ser impartida en la educación preescolar, básica y media, de conformidad con el artículo 41 de la Constitución Política.

En la capacitación a que se refiere este literal, deberán impartirse nociones básicas sobre jurisdicción de paz, mecanismos alternativos de solución de conflictos, derecho de familia, derecho laboral y contratos más usuales.

La forma en que se ha entendido y aplicado esa normatividad, coincide con las estrategias que se han planteado para abordar la formación ciudadana en la educación básica y media y, de hecho, se han propuesto una suerte de competencias ciudadanas en las discusiones que se han suscitado al respecto. No obstante, es menester tener en cuenta que dichas competencias y, sobre todo, la definición de sus indicadores para la evaluación, se constituyen en objeto de un análisis profundo en la medida en que, de algún modo, la universidad dejaría de lado su rol de espectadora de la polis y, por el contrario, la polis, en cabeza de los políticos profesionales, se erigiría como espectadora de los miembros de la academia, tras la excusa aparentemente incontestable de la calidad de la educación.

En efecto, al poder ser orientadas desde una instancia superior y exhibidas por los propios estudiantes, las competencias ciudadanas serán sin duda objeto de verificación y de evaluación y así los ingenieros, los médicos y los abogados podrán ser clasificados, no solamente por sus competencias técnicas, sino también por sus competencias ciudadanas.

Aunque se acepta que la formación ciudadana se da en múltiples y diversos escenarios, finalmente sería el Estado quien tendría la potestad y la última palabra acerca de si lo realizado en todas esas instancias diferentes a las instituciones educativas, corresponde o no con sus propias expectativas, limitadas a los juicios realizados con fundamento en un sistema al que se adscriben, "voluntariamente". Esto vulnera la autonomía 
universitaria, toda vez que el Estado podrá definir si tal o cual universidad forma bien o mal a sus estudiantes como ciudadanos y deja de lado cualquier posibilidad del ejercicio de la competencia por excelencia de la universidad, la crítica; y la de los universitarios: constituirse en miembros de una comunidad política, con sus palabras y sus actos. Que el Estado pueda calificar la calidad ciudadana de los estudiantes universitarios significa también que un actor pueda controlar el juicio que de su propia actuación hacen los espectadores. La evaluación de los indicadores de competencias ciudadanas en los jóvenes universitarios llegaría a constituirse, igualmente, en un formalismo parecido a la implementación de los cursos para la formación ciudadana en los currículos, atendiendo en nuestro medio a un mandato constitucional, pero con el agravante de los juicios del Estado sobre la calidad de los ciudadanos universitarios.

Sin perjuicio de pretender llenar los vacíos que considera heredados de la educación básica, la universidad debe constituirse en un espacio de aparición eminentemente político. Entre los temas y los problemas que han orientado a la educación para la ciudadanía en la educación superior se incluyen, de hecho, las necesidades locales de cada región y de cada país en asuntos como la pluralidad y la multiculturalidad, la exclusión y la apatía, unidos a otros también de naturaleza compleja a nivel global como los derechos humanos, el medio ambiente, las redes de información, la democratización del conocimiento y la competitividad (Bartolomé y Cabrera, 2003).

Teniendo como antecedente a la Unión Europea, las competencias ciudadanas y, de modo general, la formación ciudadana universitaria, se han ido orientando hacia el intercambio entre lo local y lo global, en el horizonte de una posible identidad global de ciudadano, en diversidad de intereses, de los que dan cuenta las palabras que discurren en el orbe internacional en los titulares de las publicaciones actuales como: identidad global, universal, cosmopolita, multicultural, transnacional, planetaria, etcétera.

\section{La formación ciudadana en la Universidad de Antioquia}

\section{Un caso}

Como se verá de manera resumida, el trabajo realizado en la Universidad de Antioquia en materia de formación ciudadana con y para su comunidad universitaria, tiene un recorrido que refleja la preocupación por el tema, que entronca, incluso, con las posibilidades del desarrollo de una ciudadanía crítica de frente a la formación para la participación consciente en el marco de las complejidades internas que vive la Universidad y los problemas sociales de su entorno. 
En aras de realizar una aproximación más fáctica a la relación educación, universidad y formación para la ciudadanía, en el acercamiento realizado específicamente a la Universidad de Antioquia por el estudio de Benjumea et al. (2015), se partió de reconocer a la Universidad como escenario complejo en el que confluyen los más diversos intereses e ideologías que se ponen en tensión. Su carácter público la hace vulnerable a problemáticas sociales tales como la polarización, la desigualdad, el tráfico y el consumo de drogas y alcohol, la violencia, la intolerancia ideológica y la apropiación del uso del espacio público, entre otras. Algunas de estas manifestaciones ponen de relieve los distintos proyectos de universidad que tienen los miembros de la comunidad académica. Justamente la heterogénea composición de esta comunidad hace - o debería hacerde la Universidad un espacio de aparición para el análisis de los asuntos de la sociedad a la que se debe, a fin de generar sentido común sobre dichos problemas y emprender líneas de acción, razón de ser de la academia.

En todo ese complejo panorama de relaciones e interacciones, hay una preocupación por la formación ciudadana de la comunidad educativa en general y de manera central de sus estudiantes, visible y expreso en diferentes trazos, normativas, proyectos y planes de desarrollo institucionales que fungen como directriz y bitácora de acción para los accionares de las distintas instancias y unidades de funcionamiento académico y administrativo. En las próximas líneas se presentará, grosso modo, un panorama general de los trazos, acciones y estrategias institucionales más visibles en la Universidad.

El Plan de Desarrollo de la Universidad de Antioquia (PD-UdeA), tanto en el periodo anterior 2006-2016: "Una universidad investigadora, innovadora y humanista al servicio de las regiones y del país" (Universidad de Antioquia, 2006), como el actual 2017-2026: "Una universidad innovadora para la transformación de los territorios" (Universidad de Antioquia, 2017), deja leer en su misión y visión conceptos y categorías que se trazan como ejes y propósitos misionales fundamentales y que se configuran en la esencia misma de la formación para la ciudadanía. En ellos se lee, cómo la Universidad, en tanto universidad pública y en ejercicio pleno de su autonomía, se compromete con la formación integral del talento humano, la formación de profesionales integrales para una paz, una convivencia, un respeto a la norma, un estar en colectivo y a un compartir; siendo líderes en el aporte a la transformación socioeconómica del país y, en la solución de los problemas regionales y nacionales, con visión universal; buscando configurar un auténtico escenario para la diversidad, el pluralismo, el diálogo de saberes y la interculturalidad.

Además, se compromete con la configuración de realidades académicas y profesionales en prospectiva de formar "individuos autónomos, conocedores de los principios éticos, responsables de sus actos, capaces de trabajar en equipo, con libre ejercicio del 
juicio y de la crítica" (Universidad de Antioquia, 2017, p. 14). Se visiona "como institución pública humanista, incluyente y democrática .... conectada con el mundo, que se transforma a sí misma y genera soluciones desde los territorios para la paz y el bienestar de una sociedad equitativa y ambientalmente sostenible" (Universidad de Antioquia, 2017 p. 5). Directrices estas que deben estar visibles en todas las iniciativas de las distintas unidades académicas y administrativas.

\section{Acciones institucionales visibles}

\section{Cátedra de formación ciudadana y constitucional}

Tal vez la estrategia más emblemática de educación para la ciudadanía en la Universidad de Antioquia, es la cátedra de formación ciudadana y constitucional creada en 1994 (Acuerdo Académico 014) para responder a la demanda de la Constitución Política de 1991. El hecho de que el curso sea dictado por abogados exclusivamente, que forme parte del plan de estudios y que sea obligatorio para todos los programas académicos, excepción hecha a los estudiantes de Derecho, señala el concepto de formación y de ciudadanía que le subyacen; el primero reducido al de instrucción cívica y el segundo a su carácter jurídico, a la espera de que el estudiante, luego de conocer la organización del Estado, los derechos, los deberes y los mecanismos de participación, se constituya en un "ciudadano activo".

Es claro que el curso en ciernes no parece llenar las expectativas de los estudiantes en cuanto a contenidos, métodos, estrategias y prácticas, tal y como se ha evidenciado en las investigaciones realizadas en la Universidad (Mesa, 2011; Pimienta et al., 2015; Sandoval et al., 2012). De hecho, las manifestaciones políticas de los estudiantes en la asamblea general - espacio donde participan para analizar diversos temas y problemas de dentro y fuera de la Universidad- marchan paralelas a los lineamientos recibidos en el curso.

Cabe aclarar que no se trata de descalificar las intencionalidades y los aportes educativos que esta cátedra pueda aportar; sin embargo, es bien claro que dicho espacio -en tanto es prácticamente el único garantizado durante el desarrollo curricular de las distintas unidades académicas para adelantar esta tarea- no puede reducirse únicamente al rastreo y a la socialización de información jurídica o legal que contiene la normativa constitucional; dejando de lado la necesidad y la esencia misma de la formación ciudadana que pone en su centro la esfera de lo político y así la constitución de la propia experiencia de participación en el espacio público. 
El análisis de la cátedra de formación ciudadana y constitucional motivó en 2011 la creación de la Comisión de Formación Ciudadana de y para la Universidad, adscrita a la Vicerrectoría de Docencia y conformada, además, por otros docentes de las áreas de la salud, ciencias sociales y humanas, ciencias exactas y naturales, y de unidades académicas, como las facultades de ingeniería, derecho y ciencias políticas, configurando, así, un equipo de sujetos universitarios con experiencia en la cátedra.

Los análisis allí planteados han discurrido alrededor de la esencia misma de esta cátedra y el análisis de su resignificación; además de la comprensión misma y redefinición que la Universidad tenía en torno a conceptos como ciudadanía, formación y educación. Igualmente, esta comisión que sufrió algunas reconfiguraciones en el tiempo, abordó otros lineamientos que buscaron ir más allá del análisis del curso y de los conceptos involucrados, para pensar estrategias que fortalecieran las discusiones y las acciones en torno a esta proyectiva en la Universidad y avanzar así en la tarea de formular la política al respecto, labor nada fácil si se tienen en cuenta las circunstancias propias de la Universidad, cuyo ambiente se ha caldeado de modo singular en los últimos años debido a múltiples problemas que ha tenido que sortear, en temas tan sensibles como la función social y el futuro mismo de la educación superior y, de manera especial, de la educación pública.

Teniendo presente la realidad nacional y particularmente la de la Universidad, la Comisión ha ido avanzando hasta presentar entre otras alternativas, el enriquecimiento de los escenarios de encuentro, de debate y deliberación, junto con la conservación y el desarrollo de la información y la difusión acerca de la Carta Política, nervio del curso de formación ciudadana y constitucional. Sin embargo, no es suficiente y debe ambicionarse que cada miembro de la comunidad educativa, cada dependencia, se involucre y se piense desde su responsabilidad para generar otras posibilidades del cómo nutrir lo que hay, cómo articular los procesos, cómo vivir la cotidianidad universitaria y cómo resignificar y proyectar estos en las comunidades que dichos actores habitan.

La Comisión, más allá de los análisis académicos y políticos, ha quedado con el reto de desarrollar una política de formación ciudadana en la Universidad, lo cual entraña, no solo un concepto amplio de esta, sino también el análisis de la Universidad como espacio público, como espacio de aparición de los iguales para exponer tesis, pensamientos e ideas, muchas veces antagónicas, con la formidable tarea de que dicho antagonismo se convierta en agonismo; es decir, en un espacio en el que no se trata de eliminar o vencer al otro, sino de compartir puntos de vista para la construcción y la conservación de los espacios en que nos "aparecemos". 
En la actualidad, 2020, es visible en el portal universitario de la Universidad, un espacio denominado "Formación ciudadana y participación universitaria", donde se hace un llamado a la participación en tanto derecho individual y colectivo de la vida institucional, expreso en mecanismos constitucionales acogidos por leyes y normas universitarias (Estatuto General de la UdeA, artículo 18). Llamado que insta a "la activación política de los estamentos" con la participación en los "organismos colegiados"; con la intención de cualificar procesos de toma de decisiones por medio del diálogo y la deliberación entre los diferentes actores universitarios, en "espacios de interlocución que consideren posturas divergentes y legítimas de las demandas colectivas"5.

\section{Bienestar universitario}

El sistema de bienestar universitario de la Universidad de Antioquia acompaña con sus programas y acciones a los miembros de la comunidad académica en sus distintas dinámicas de vida universitaria. Ha enfocado su tarea a aspectos que abogan por la calidad de vida, formación integral y sentido de comunidad desarrollados mediante diferentes programas y servicios que se planean en perspectiva de desarrollo humano, promoción de la salud y prevención de la enfermedad, además de la práctica deportiva, entre otros.

El plan de acción del sistema de bienestar vigente, trazado a la luz de los PD-UdeA de los dos últimos periodos, establece claramente sectores estratégicos con elementos inherentes a la formación ciudadana, enfocando su ejercicio en ejes misionales como: el sentido de comunidad, la calidad de vida y la formación ciudadana; esta última dirigida a favorecer, en los diferentes actores de la Universidad, diversas formas de vivir, elaborar y promover actitudes y aptitudes, enmarcados en el respeto a la diferencia y la diversidad. En la actualidad dirige su quehacer a estrategias trazadas en perspectiva de equidad e igualdad de oportunidades, al desarrollo de hábitos y estilos de vida saludables, de habilidades personales y para la vida profesional; $y$, en procesos de educación y fomento para la participación y vida universitaria. Este último, haciendo explícitos temas como educación en y sobre lo público, fomento de la participación responsable, promoción de la convivencia, la ciudadanía y el respeto por el entorno.

Es de resaltar que el sistema de bienestar universitario de la Universidad se ha centrado en promover estrategias desde el reconocimiento de la realidad socioeconómica de los estudiantes, a partir de lo cual se han desplegado diversas acciones y servicios para responder a las aspiraciones de la comunidad universitaria, favoreciendo la permanencia y el desempeño académico, reduciendo, además, los índices de deserción. Pese a

5. http://www.udea.edu.co/wps/portal/udea/web/inicio/institucional/formacion-ciudadana-participacion-universitaria 
que en la época de los años de 1990 la intervención desde el bienestar universitario se visualizó con un claro enfoque de promoción y prevención asistencialista, en la actualidad se observa un matiz diferente que alienta una perspectiva más formadora, en tanto proyecta una visión integral que establece clara relación con la docencia, la investigación, la extensión y la gestión de la Universidad.

Desde esta visión integral y analizando el carácter asistencialista que fue tomando el apoyo socioeconómico brindado a los estudiantes, se ha promovido un enfoque de participación ciudadana y convivencia con la implementación desde 2014 de programas como: "Tejiendo redes", "Arte y cultura para el bienestar" y, en especial, el programa "Promotores para el bienestar", cuyas líneas estratégicas, diversidad y cultura, y cultura Universidad de Antioquia, buscan el empoderamiento de los estudiantes como gestores de su bienestar y el fortalecimiento del ejercicio ciudadano, reconociendo a toda la comunidad universitaria como actores, gestores y promotores en acciones de liderazgo, participación y reconocimiento de su papel como agentes de cambio.

\section{Otras realidades de formación ciudadana en la Universidad de Antioquia}

\section{Aquello no formal que circula en la cotidianidad del campus universitario}

Partiendo del reconocimiento de la sana intencionalidad, reflejada en los discursos de las diferentes unidades académicas, que dicen acoger la directriz central de reflejar en sus respectivos planes de acción y programas académicos sus propias estrategias para impulsar estas iniciativas, $y$, sin desconocer que en realidad se intenta responder a este reto desde aspectos que se hacen visibles en la gran mayoría de los currículos, con programación de cursos y actividades con énfasis en el desarrollo humano y la proyección social, en los cuales se exploran aspectos éticos y algunos valores ciudadanos, sin que se aluda necesariamente de modo específico a estos, se hacen igualmente visibles y quizás más fuertes otras realidades.

Y así, a pesar de lo prescrito, se observa igualmente cómo en la cotidianidad discurre el proceso académico de las distintas áreas de conocimiento que en sus dinámicas reflejan que su interés esencial, "en el paso por la universidad", es el desarrollo de los contenidos disciplinarios, pues sustentan la necesidad de dar elementos para responder a los retos de la competitividad del medio; y aquellos tan necesarios y esperados espacios para la formación de un juicio reflexivo no son claros, visibles y hasta podría decirse que son poco considerados, aunque en los perfiles de formación, misiones y otros documentos que soportan las estructuras curriculares se trazan como elementos propositivos de su razón de ser. 
La Universidad de Antioquia, en términos de formación ciudadana, sobrepasa lo prescriptivo porque constituye de suyo una multiplicidad y pluralidad de espacios, costumbres y poblaciones, configurándose así una diversidad de espacios de aparición, muchos de los cuales escapan a la intencionalidad de la educación y al control por medio del currículo formal, laboratorio en el cual se pueden vivir, permear y analizar singularidades de la educación y la formación en relación con la ciudadanía como campo emergente en la educación superior; asimismo, puede verse como un inigualable nicho de consolidación de una ciudadanía necesaria y deseada, en la medida en que no se pretende una formación intencionada sino que esta, es un dándose que se sucede en lo que Arendt (2008) llamaría el "entre" o la reunión de los ciudadanos, cuya empatía no reside en una característica especial; por ejemplo, un credo, una ideología, una raza, una etnia o un presunto mejor origen que los cohesiona, sino que se reconocen como seres humanos que gozan del privilegio de escucharse unos a otros para tener una perspectiva mucho más amplia del tema que se trata e, incluso, para cambiar el propio punto de vista.

Dentro del currículo informal se contemplan para la Universidad los mecanismos de participación en diferentes instancias de gobierno tales como la representación de docentes y estudiantes en los consejos Superior, Académico, o en el de las unidades académicas; y en otras cuyo carácter es de asesoría, como sucede, por ejemplo, con los comités de currículo y de carrera. Si bien estos espacios deberían garantizar a cada actor el derecho, pero también el deber a un modo de participación activa en el proyecto de universidad; se observa en los diferentes estamentos luchas de poder y afanes por ganancias particulares que van en contravía de lo esperado, provocando descrédito en estos órganos de representación, indiferencia y hasta rechazo por las acciones que ellos promueven.

Regularmente, desde las vicerrectorías o desde las unidades académicas emergen iniciativas que no siempre alcanzan una sostenibilidad en el tiempo, en tanto no se gestan redes de apoyo, divulgación, motivación y compromiso de distintos actores y dependencias; y en la mayoría de los casos se quedan en un repetir intentos desde distintos frentes, o en el lejano eco de un instante de inspiración de un grupo o dependencia que se expuso y se atrevió a intentarlo.

A modo de ejemplo, puede traerse acá como un primer planteamiento de posibilidad sobre aquello no formal que circula en la cotidianidad del campus universitario, lo que viene aconteciendo hace varios años - segundo semestre de 2012-, en y desde una

6. Con el "entre" Arendt hace referencia a la reunión de los ciudadanos sin que tengan necesariamente filiaciones de tipo social, étnico, cultural, religioso o político, sino alrededor de los asuntos de la comunidad. 
unidad académica de la Universidad ${ }^{7}$, donde en una búsqueda por encontrar una ruta que permitiera asir, de forma fáctica, procesos de formación y motivación en y para la participación, emergió la estrategia Re-creándonos - de la cual se ha hablado en párrafos anteriores-, iniciativa surgida de un grupo de estudiantes motivados y liderados por una docente, bajo el pregón de que "en el paso por la universidad" deberían suceder más cosas que lo que acontece en el aula y está inscrito claramente en el currículo del programa de formación; lo que devino desde entonces, en unos espacios de "disertación" sobre temáticas emergentes de los acontecimientos que se suceden en la Universidad, y que en palabras de los participantes "poco sabían": "las acciones violentas en y de la U", "la situación actual de la Universidad”, "en qué consiste el proyecto de universidad pública”, "cuál es el rol de la educación en los procesos de transformación social”, "los modos en que los estudiantes de la U participan en las problemáticas del país”, "¿se tienen que hacer manifestaciones violentas para que nos escuchen?", “¿cuál es la responsabilidad social de la universidad en esos asuntos”, “¿qué significa ser sujeto universitario”, etcétera.

Esta disertación prosperó y se fortaleció con el convencimiento de que la realidad sociopolítica de la Universidad, en tanto universidad pública, es un nicho completamente fértil para potenciar una iniciativa necesaria que permita conocer, entender, vivir y participar activamente en la consolidación del proyecto de universidad. De este modo, las propuestas, las acciones y las proyectivas de Re-creándonos siguen fundamentándose en el interés de repensarnos, resignificarnos y recrearnos como seres coparticipes de un proyecto sociocultural donde se reconozca que las prácticas motrices lúdico-deportivas, recreativas, artísticas y culturales, también son prácticas políticas.

Es factible, entonces, encontrar, en el transcurrir de la cotidianidad por espacios universitarios, otras propuestas, que como esta - sin necesidad de partir de directrices institucionales - se gestan como espacios de creación del bienestar común, prosperan bajo un esfuerzo de trabajo mancomunado y nos forman como sujetos corresponsables; formación que se da en la interacción con el otro, los otros y con lo otro. Espacio donde interesa más el ser que el hacer, sin importar las diferencias tanto socioculturales como naturales; donde nos reconocemos como un legítimo otro y respetamos ese otro como diferente y a la vez igual a mí.

La Universidad de Antioquia, en tanto universidad pública, reclama con urgencia el reconocimiento de estos "otros" espacios donde los jóvenes puedan expresar y potenciar iniciativas múltiples, variadas. Nichos indiscutiblemente fértiles para discernir en torno a preocupaciones de la universidad y de la sociedad; estrategias innovadoras

7. Instituto Universitario de Educación Física y Deportes de la Universidad de Antioquia (IUEFD). 
que desborden la reflexión como contenido y favorezcan la inclusión, la participación y la formación del juicio crítico reflexivo, poniendo en juego la acción fundamentada para ser, conocer, decidir y participar activamente de lo público - ejercicio ciudadano-.

\section{Para no concluir, más bien, reflexiones para seguir}

Si bien en torno al tema sobre educación para la ciudadanía y la formación ciudadana en la universidad no se encuentra un amplio despliegue de reflexiones e investigaciones, no obstante, como puede observarse en la mirada panorámica acá presentada, el tema ha ido tomando el carácter de emergente en las agendas académico-administrativas e investigativas de las universidades en los últimos años, mostrando aristas discursivas y reflexivas con respecto al papel de la universidad como escenario para este tipo de formación y el rol de los distintos actores universitarios en este proceso formativo.

Cabe resaltar que un tema como la formación ciudadana en el espacio universitario debe superar el lugar de lo prescrito a nivel institucional y constituirse en asunto que discurra sin fronteras de tiempo y espacio dentro del campus, configurarse en una esencia que tome formas múltiples, plurales, diversas, tantas como sean posibles; acciones que como prismas dibujen variadas rutas y engendren tantas posibilidades como actores haya.

Entonces, para no concluir, más bien para dejar abiertas unas apuestas, unos cuantos trazos y seguir atentos y dispuestos a la disertación, al debate -dado que la complejidad de esta temática nunca podrá abordarse en su amplitud desde un texto, independiente de su extensión-, se abren reflexiones que se hilen y entretejan en torno al eje central de la responsabilidad de la universidad para la formación política de los universitarios. En particular la Universidad de Antioquia define la responsabilidad social:

[...] como el desarrollo de autorreflexión sobre las dinámicas de la Universidad, basadas en la ética como principio, la definición de sus grupos de interés y las relaciones que estos establecen con la Institución. Se establece el seguimiento y la evaluación del dinamismo de las relaciones y la reciprocidad de las acciones de la Universidad y sus actores, partiendo del reconocimiento del otro, humano y su territorio, como el objeto de sus acciones y fines. (Universidad de Antioquia, 2019)8

En esa perspectiva, nos seguiremos preguntando cómo seguir tejiendo reflexiones en torno a todo aquello que subyace y circula en esa interacción inherente que hay entre universidad/conocimiento -sociedad-sujeto universitario-ciudadano...- y sobre

8. Acuerdo Superior 463, artículo 2: del 29 de octubre de 2019 que establece y adopta la política de la responsabilidad social universitaria de la Universidad de Antioquia. 
otros modos que nos permitan superar las brechas y el vacío existentes entre la teoría y la práctica del mundo de la academia y nos permitan asir, de manera fáctica, la relación formación académica con la formación para la vida.

Se seguirá apostando por una educación centrada en el desarrollo de las potencialidades humanas en la medida en que el interés pedagógico trabaje desde la autorrealización del sujeto; es decir, hacerse sujeto mediante múltiples formas expresivas, donde además de propiciar el desarrollo de capacidades, también se posibilite el hablar y expresar desde el sentimiento, la experiencia, la emoción, la motivación y desde la ética individual y colectiva, lo cual está íntimamente relacionado con la responsabilidad y corresponsabilidad consigo mismo, con los demás y con los entornos; es decir, una articulación ética de la producción del conocimiento, con la realidad del sujeto: el sentir, el querer, el hacer, el pensar, el actuar y el crear.

Se insistirá en la necesidad de reconocer que, como miembros de la comunidad universitaria, somos sujetos de derechos, pero también de deberes y, en la urgencia de comprometernos con ello, entendiendo, como lo expresa Hannah Arendt, que en un espacio de aparición siempre hay un actor y un espectador, y para este caso, es dejar de ser espectadores pasivos e indiferentes y consolidarnos como actores partícipes de su desarrollo.

\section{Referencias}

[1] Alvarado, S., Ospina, H., Botero, P. y Muñoz, G. (2008). Las tramas de la subjetividad política y los desafíos a la formación ciudadana en jóvenes. Revista Argentina de Sociología, 6(11), 19-43.

[2] Arendt, H. (2008). La promesa de la política. Paidós.

[3] Arendt, H. (2007). Responsabilidad y juicio. Paidós.

[4] Bartolomé Pina, M. y Cabrera Rodríguez, F. (2003). Sociedad multicultural y ciudadanía: hacia una sociedad y ciudadanía interculturales. Revista de educación, 1, 33-56. http:// www.educacionyfp.gob.es/revista-de-educacion/numeros-revista-educacion/ numeros-anteriores/2003/re2003/re2003-03.html

[5] Benjumea, M., Bustamante, S., Hernández, P. y Mesa, A. (2015). La Formación Ciudadana en el contexto universitario de la UdeA -análisis de estrategias para su resignificación y potencialización desde la cotidianidad en el campus [Proyecto de investigación CODI]. Universidad de Antioquia (2013-2015).

[6] Bolívar, A. (2007). Educación para la ciudadanía. Algo más que una asignatura. Graó.

[7] Cuello, M. (2010). Influencia de la formación de grado y la pertenencia institucional universitaria sobre la comprensión de temas políticos y la formación ciudadana. Revista Electrónica de Psicología Política, 8(22). http://www.psicopol.unsl.edu.ar/abril2010_Nota3.pdf 
[8] Del Basto, L. (2006). Relación universidad-sociedad civil en el ámbito de lo público. Una reflexión necesaria. Rhec. Revista Historia de la Educación Colombiana, 9, 93-109. https://revistas.udenar.edu.co/index.php/rhec/article/view/1075

[9] Del Río, N. y Rizzini, I. (2010). Participación político-social juvenil en dos contextos urbanos latinoamericanos: Río de Janeiro y Ciudad de México. En O. Roldán Vargas (coord.), Niñez y juventud latinoamericanas. Experiencias de relacionamiento y acción colectiva (pp. 75-104). Fundación CINDE.

[10] Englund, T. (2002). Higher Education, Democracy and Citizenship - the Democratic Potential of the University? Studies in Philosophy and Education, 21(4/5), 281-287. https://doi.org/10.1023/A:1019840006193

[11] Eyler, J., Giles Jr, D. E. y Braxton, J. (1997). The Impact of Service-Learning on College Students. Michigan Journal of Community Service Learning, 4(1), 5-15. http://hdl. handle.net/2027/spo.3239521.0004.101

[12] Haste, H. y Hogan, A. (2006). Beyond Conventional Civic Participation, Beyond the Moral-Political Divide: Young People and Contemporary Debates about Citizenship. Journal of Moral Education, 35(4), 473-493. https://doi. org/10.1080/03057240601012238

[13] Lozano, M. y Alvarado, S. (2011). Juicios, discursos y acción política en grupos de jóvenes estudiantes universitarios de Bogotá. Revista Latinoamericana de Ciencias Sociales, Niñez y Juventud, 1(9), 101-113. http://revistaumanizales.cinde.org.co/rlcsnj/ index.php/Revista-Latinoamericana/article/view/346

[14] Magendzo, A. (2004). Formación ciudadana. Cooperativa Editorial Magisterio.

[15] Marshall, T. y Bottomore, T. (2005 [1950]). Ciudadanía y clase social. Losada.

[16] Martínez, M. (2006). Formación ciudadana y educación superior. Revista Iberoamericana de Educación, 42, 85-102.

[17] Mesa, A. (2011). El juicio de los espectadores de Hannah Arendt: entre la educación para la ciudadanía y la formación ciudadana en la universidad [Tesis doctoral, Universidad de Antioquia].

[18] Mesa, A. y Benjumea, M. (2011). La educación para la ciudadanía en la educación superior. Uni-Pluriversidad, 11(1). 85-96. https://revistas.udea.edu.co/index.php/ unip/article/view/10581

[19] Pimienta, A., Quiroz, R., Mesa, A. y Piedrahita, L. (2015). Constitución de subjetividades políticas desde la formación ciudadana y el proyecto político de universidad. Análisis del discurso de los estudiantes universitarios [Proyecto de investigación CODI]. Universidad de Antioquia.

[20] Quintero, M. (2006). Justificaciones de jóvenes universitarios y jóvenes desplazados acerca de la concepción de justicia [tesis doctoral, Universidad de Manizales - CINDE]. 
[21] República de Colombia. (1991). Constitución Política de Colombia. Leyer.

[22] República de Colombia. (2006, 23 de enero). Por la cual se modifica el artículo 14 de la Ley 115 de 1994 [Ley 1013 de 2006]. Diario Oficial No. 46.160. https://www. funcionpublica.gov.co/eva/gestornormativo/norma.php?i=18877

[23] Sandoval, C., Villa, M., Mesa, A. y Benjumea, M. (2012). Representaciones sociales, expresiones de participación, razonamiento social y prácticas educativas, relacionadas con la formación ciudadana en el contexto universitario: un análisis del sentido y condiciones de posibilidad, de un proyecto de formación ciudadana en la educación superior [Proyecto de investigación CODI]. Universidad de Antioquia.

[24] Santa Cruz, L. (2004). Reflexiones críticas en torno a la formación ciudadana en la institución escolar. Revista Docencia, 23, 36-47.

[25] Santana, C., Bogoya, N. y Rojas, G. (2005). Valores ciudadanos y democráticos: una perspectiva en la formación universitaria. Revista de Estudios y Experiencias en Educación, 4 (8), 25-38. http://www.rexe.cl/ojournal/index.php/rexe/article/view/208

[26] Tonon, G. (2009). La universidad como escenario de construcción de ciudadanía: percepción de jóvenes estudiantes de la carrera de Ciencia Política de la Universidad Nacional de la Matanza, Argentina. En O. Roldán Vargas (coord.), Niñez y juventud latinoamericanas. Experiencias de relacionamiento y acción colectiva (pp. 51-74). Fundación CINDE.

[27] Universidad de Antioquia. (1994). Estatuto general de la Universidad de Antioquia. http:// portal.udea.edu.co/wps/wcm/connect/udea/ad4c0fd3-3486-481a-be87-8020a4652 b85/1.+Estatuto+General+Acuerdo+Superior+1+de+1994.pdf?MOD=AJPERES

[28] Universidad de Antioquia. (2006). Plan de desarrollo 2006-2016. Una universidad investigadora, innovadora y humanista al servicio de las regiones y del país. http://www. udea.edu.co/plandedesarrollo

[29] Universidad de Antioquia. (2017). Plan de desarrollo 2017-2026. Una universidad innovadora para la transformación de los territorios. http://www.udea.edu.co/plandedesarrollo

[30] Universidad de Antioquia. (2019, 29 de octubre). La política de la responsabilidad social universitaria de la UdeA. Acuerdo Superior 463.

[31] Warwick, P. (2008). The Development of Apt Citizenship Education through Listening to Young People's Voices. Educational Action Research, 16(3), 321-333. https://doi.org/10.1080/09650790802260232

[32] Wilhite, S. y Silver, P. (2005). A False Dichotomy for Higher Education: Educating Citizens vs. Educating Technicians. National Civic Review, 94(2), 46-54. https://doi. org/10.1002/ncr.94 